\title{
Decoherence from dipolar interspin interactions in molecular spin qubits
}

\author{
Luis Escalera-Moreno, Alejandro Gaita-Ariño, ${ }^{*}$ and Eugenio Coronado \\ Unidad de Investigación en Materiales Moleculares - Instituto de Ciencia Molecular, University of Valencia, \\ Calle catedrático José Beltrán 2, 46980 Paterna, Spain
}

(Received 27 July 2018; published 13 August 2019)

\begin{abstract}
The realization of spin-based logical gates crucially depends on magnetically coupled spin qubits. Thus, understanding decoherence when spin qubits are in close proximity will become a roadblock to overcome. Herein, we propose a method free of fitting parameters to evaluate the qubit phase memory time $T_{m}$ in samples with high electron spin concentrations. The method is based on a model aimed to estimate magnetic nuclear decoherence [P. C. E. Stamp and I. S. Tupitsyn, Phys. Rev. B 69, 014401 (2004)]. It is applied to a ground-spin $J=8$ magnetic molecule 1 displaying atomic clock transitions, namely $\left[\mathrm{Ho}^{\mathrm{III}}\left(\mathrm{W}_{5} \mathrm{O}_{18}\right)_{2}\right]^{9^{-}}$, which remarkably increase $T_{m}$ at unusually high electron-spin concentrations. Our approach unveils the causes that limit the coherence reached at the clock transitions in challenging systems such as $\mathbf{1}$, where recent models fail.
\end{abstract}

DOI: 10.1103/PhysRevB.100.064405

\section{INTRODUCTION}

Spin qubits are promising candidates as building blocks for storage and computation of quantum information [1-4]. These minimum-information units are encoded in energy levels of magnetic systems, and can be probed via different techniques such as electron paramagnetic resonance (EPR). The good performance of a spin qubit relies on its ability to keep the quantum information saved in it intact for a long enough time. Nevertheless, as it happens in other physical qubits $[5,6]$, spin qubits are affected by uncontrolled environmental interactions which tend to destroy the stored information. The nature of this phenomenon - called quantum decoherence-depends on the qubit and on the experimental conditions. Two main mechanisms can collapse the quantum information saved in a spin qubit. These are vibrational decoherence-induced by the coupling of the spin with lattice vibrations [7-10]-and magnetic decoherence-caused by the dipolar interaction of the spin qubit with surrounding nuclear and electron spins [11]. These are the main decoherence mechanisms to focus on in molecular spin qubits, and the key goal will be to suppress them as much as possible to keep quantum information intact.

The standard method employed to suppress the second mechanism consists of (i) placing qubit carriers in nuclearspin-free environments and (ii) diluting these carriers among their diamagnetic analogs, where the magnetic entity is replaced by a diamagnetic one [12]. Optimized combinations of these approximations remarkably increase the phase memory time $T_{m}$ [3], which is a figure of merit determined by EPR that characterizes how long a spin qubit can keep quantum information safe. Nevertheless, isolation of qubits is impractical at the stage of device design, since the implementation of logical gates for quantum algorithms requires communication among close qubits [13-15].

\footnotetext{
*alejandro.gaita@uv.es
}

A strategy to overcome this drawback is operating at atomic clock transitions, also known as zero first-order Zeeman (ZEFOZ) shifts (Fig. 1) [16-19]. These are avoided energy crossings in which Zeeman effect vanishes up to first order, making qubit coherence become remarkably insensitive to surrounding magnetic noise. Within moleculebased spin qubits, this method was recently demonstrated for the first time, and it allowed reaching long $T_{m}$ values at unusual high electron-spin concentrations [4]. Besides, molecular-spin qubits operating at atomic clock transitions are also potential candidates to implement logical gates as a part of scalable architectures [20]; see Supplemental Material 6 [21].

Herein, we aim to unveil the key factors limiting the phase memory time in spin qubits surrounded by high electron-spin

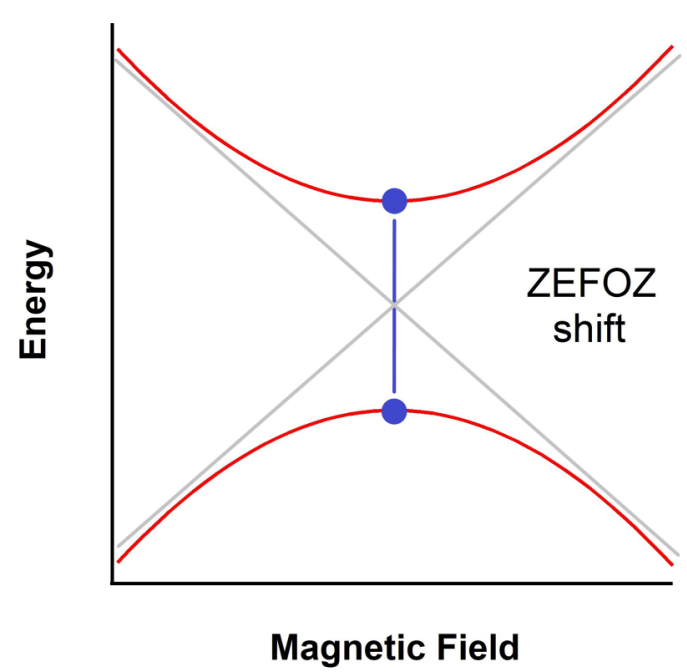

FIG. 1. Schematic representation of a ZEFOZ shift in blue at an avoided crossing between two energy levels in red. Note the vanishing slopes at the ZEFOZ field. 

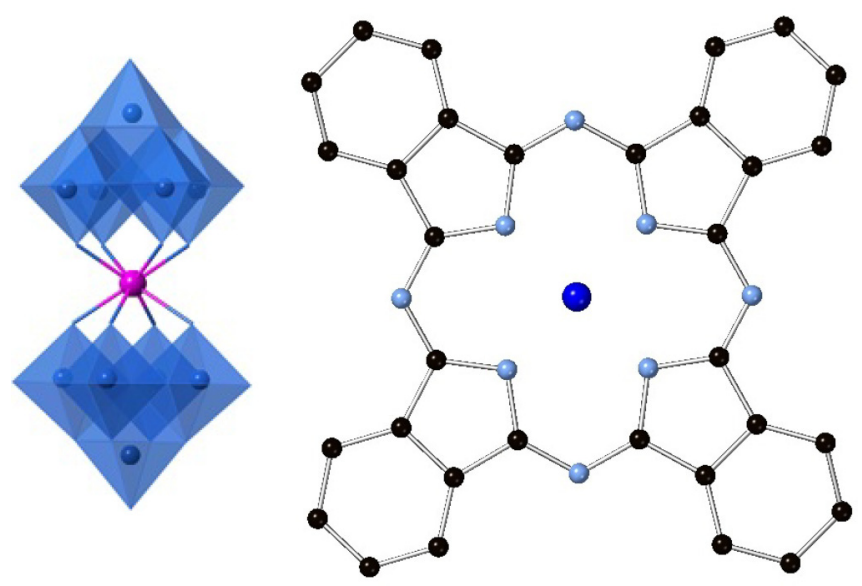

FIG. 2. Left: $1\left[\mathrm{Ho}^{\mathrm{III}}\left(\mathrm{W}_{5} \mathrm{O}_{18}\right)_{2}\right]^{9-}$ (abbreviated as $\left.\mathrm{HoW}_{10}\right)$; blue spheres: tungsten, magenta sphere: holmium, polyhedron vertexes: oxygen. Right: $2 \mathrm{C}_{32} \mathrm{H}_{16} \mathrm{~N}_{8} \mathrm{Cu}^{\mathrm{II}}$ (abbreviated as $\mathrm{CuPc}$ ); black: carbon, light blue: nitrogen, dark blue: copper. Hydrogen atoms in $\mathbf{2}$ are omitted for clarity.

concentrations. The target system is a single crystal composed of many identical copies of a $\mathrm{Ho}^{\mathrm{III}}$-based molecular spin qubit, 1 (Fig. 2), displaying ZEFOZ shifts [4]. 1 is a magnetic molecule composed of a single $\mathrm{Ho}^{\mathrm{III}}$ ion that is coordinated by two polyoxometalate ligands $\left[\left(\mathrm{W}_{5} \mathrm{O}_{18}\right)_{2}\right]^{12-}$. Four peculiar narrow regions appear in the experimental $T_{m}$ magnetic-field dependence of $\mathbf{1}$, where the phase memory time sharply increases up to a maximum value (Fig. 3). To understand the origin of this limiting value, we firstly applied a recent model that satisfactorily explained the experimental $T_{m}$ evolution of a molecular spin-1/2 qubit, 2 (Fig. 2) at increasing electron-spin concentration [26]. However, this model overestimates the experimental $T_{m}$ top values reached at the ZEFOZ fields in $\mathbf{1}$ as we will see below. This motivates us to propose a method free of fitting parameters to evaluate $T_{m}$, which relies on a model aimed to estimate magnetic nuclear decoherence [27]. Our method does successfully reproduce the experimental height of $T_{m}$ at the ZEFOZ fields in 1 (Fig. 3) and works provided instantaneous diffusion is the limiting decoherence mechanism, which requires high electron-spin concentrations $[28,29]$. Thus, the effect of other mechanisms on $T_{m}$ are beyond the scope of the present work. As a further test, we check that our method, which is independent of that reported in Ref. [26], is also able to reproduce the experimental $T_{m}$ evolution of 2 at increasing electron-spin concentration (Fig. 4).

\section{METHOD}

Nonspecialist readers may want to avoid this part of the paper and go directly to Sec. III, as herein we are providing the technical details of the derivation of our method. The starting point of our method is a model developed elsewhere whose calculation of the phase memory time $T_{m}$ from the off-diagonal element in the qubit-reduced density matrix is made in terms of a path integral over pairs of qubit trajectories [27]. Once this matrix element is calculated, it is possible to employ standard techniques of the spin-boson model to derive $T_{m}$, and a dephasing rate $\gamma$ is defined as $\gamma=2 \hbar / T_{m} \Delta, \Delta$ being the gap energy between the two spin states of the qubit $[11,27]$.

From the derivation of this model it follows that the mentioned matrix element decays as a single exponential $\exp \left(-\tau / T_{m}\right)$ with time $\tau$. It is appropriate since our method is to be employed in an experimental regime where instantaneous diffusion-high electron-spin concentrationsdominates as a dephasing mechanism, and this mechanism is characterized by a stretching factor $x=1$ in EPR experiments $[4,26,28,29]$. In these experiments, the in-plane magnetization $M_{\perp}(\tau)$ of the sample—proportional to the spin-echo signalis determined as a function of time $\tau$ [28]. Then, this time evolution is fitted to the generalized exponential $M_{\perp}(\tau)=$ $\left(M_{\perp}(0)-M_{\perp}(\infty)\right) \exp \left(-\left(\tau / T_{m}\right)^{x}\right)+M_{\perp}(\infty)$ to extract both $T_{m}$ and $x$.

Our method is applied to molecules where a single magnetic metal ion encoding the spin qubit is coordinated by a set of ligands. In these molecules, the spin anisotropy produced in the metal ion by the ligand coordination will be described by the following crystal-field Hamiltonian:

$$
\hat{H}=\sum_{k=2,4,6} \sum_{q=-k}^{+k} B_{k}^{q} \hat{O}_{k}^{q}+\overrightarrow{\hat{I}} \cdot \boldsymbol{A} \cdot \overrightarrow{\hat{J}}+\mu_{B} \vec{B} \cdot g \cdot \overrightarrow{\hat{J}}
$$

In Eq. (1), $B_{k}^{q}$ are the crystal-field parameters, $\hat{O}_{k}^{q}$ are the extended Stevens operators [30], $J$ and $I$ are the ground electron-and nuclear-spin quantum numbers of the magnetic metal ion, respectively, $g$ is the free-ion Landé factor, $\mu_{B}$ is the Bohr magneton, and $\vec{B}$ is the applied magnetic field. The model which our method relies on estimates the dephasing rate $\gamma_{n}$ due to the nuclear-spin bath [27]. We now adapt this model as what we want herein is to estimate a dephasing rate $\gamma_{e}$ due to the electron-spin bath. Thus, by assuming additive rates [11,27], it is possible to determine a collective phase memory time as $T_{m}^{n+e}=2 \hbar /\left[\left(\gamma_{n}+\gamma_{e}\right) \Delta\right]$.

Following, it is shown how we proceed to calculate the dephasing rate $\gamma_{e}$. The key step is to relate $\gamma_{n}$ and $\gamma_{e}$ with the nuclear $E_{n}$ and electron $E_{e}$ contributions to the echo line half-width. Under the so-called highfield regime, which means $E_{n} \ll \Delta, E_{e} \ll \Delta$, these rates can be calculated perturbatively up to second order resulting in $\gamma_{n}=2\left(E_{n} / \Delta\right)^{2}, \gamma_{e}=2\left(E_{e} / \Delta\right)^{2}[11,26,27]$. The range of working nuclear- and electron-spin concentrations quite often lies inside this regime [11,26,27], hence $T_{m}^{n+e}=\hbar \Delta /\left(E_{n}^{2}+E_{e}^{2}\right)$. As mentioned above, $E_{n}$ was derived elsewhere [27], and now the main goal for us is to calculate $E_{e}$.

Firstly, one considers the magnetic field under the dipolar approximation $\overrightarrow{B_{j}}$ that a given magnetic metal ion $j$ generates at the position of another given magnetic metal ion $k$ :

$$
\vec{B}_{j}\left(\overrightarrow{r_{j k}}\right)=\frac{\mu_{0}}{4 \pi} \frac{1}{r_{j k}^{5}}\left(3\left(\overrightarrow{m_{j}} \cdot \overrightarrow{r_{j k}}\right) \overrightarrow{r_{j k}}-r_{j k}^{2} \vec{m}_{j}\right) .
$$

The vector connecting these two ions is $\overrightarrow{r_{j k}}$, with a magnitude $r_{j k}$, and their magnetic moments are $\vec{m}_{j}$ and $\vec{m}_{k}$. This allows 
calculating their dipolar interaction energy as

$$
E_{j k}=-\overrightarrow{m_{k}} \cdot \overrightarrow{B_{j}}\left(\overrightarrow{r_{j k}}\right)=\frac{\mu_{0}}{4 \pi} \frac{1}{r_{j k}^{3}}\left(\overrightarrow{m_{j}}-\frac{3\left(\vec{m}_{j} \cdot \overrightarrow{r_{j k}}\right)}{r_{j k}^{2}} \overrightarrow{r_{j k}}\right) \cdot \vec{m}_{k}
$$

The diagonalization of the crystal-field Hamiltonian in Eq. (1) provides the relevant $(2 J+1)(2 I+1)$ energies and wave functions $\left\{E_{l},\left|\psi_{l}\right\rangle\right\}_{l=1, \ldots,(2 J+1)(2 I+1)}$. The wave functions are expressed with complex coefficients $c(l)_{i}^{n}$ in the basis set
$\left\{\left|m_{J}, m_{I}\right\rangle\right\}_{m_{J}=-J, \ldots, J ; m_{I}=-I, \ldots, I}$ of the $m_{J}$ and $m_{I}$ projections as

$$
\begin{aligned}
\left|\psi_{l}\right\rangle= & \sum_{n=1}^{2 J+1} \sum_{i=(n-1)(2 I+1)+1}^{n(2 I+1)} c(l)_{i}^{n} \mid n-1-J, i-(n-1) \\
& \times(2 I+1)-1-I\rangle .
\end{aligned}
$$

We need now to calculate $\left\{\left\langle\hat{J}_{\alpha}^{2}\right\rangle_{l}=\left\langle\psi_{l}\left|\hat{J}_{\alpha}^{2}\right| \psi_{l}\right\rangle\right\}_{l=1, \ldots,(2 J+1)}$ $(2 I+1) ; \alpha=x, y, z$, the expectation values of each component $(\alpha=$ $x, y, z)$ of the square electron-spin operator $\hat{J}^{2}$. After some

algebra, one obtains

$$
\begin{gathered}
\left\langle\hat{J}_{z}^{2}\right\rangle_{l}=\sum_{n=1}^{2 J+1}(n-1-J)^{2} \sum_{i=(n-1)(2 I+1)+1}^{n(2 I+1)}\left|c(l)_{i}^{n}\right|^{2}, \\
\left\langle\hat{J}_{x}^{2}\right\rangle_{l}=\frac{1}{4}\left\{2 J(J+1)-2\left\langle\hat{J}_{z}^{2}\right\rangle_{l}+\sum_{n=1}^{2 J-1}(J(J+1)-(n-1-J)(n-J))^{1 / 2}(J(J+1)-(n+1-J)(n-J))^{1 / 2}\right. \\
\left.\ldots \sum_{i=(n-1)(2 I+1)+1}^{n(2 I+1)}\left[\left(c(l)_{i+2(2 I+1)}^{n+2}\right)^{*} c(l)_{i}^{n}+\left(c(l)_{i}^{n}\right)^{*} c(l)_{i+2(2 I+1)}^{n+2}\right]\right\}, \\
\left\langle\hat{J}_{y}^{2}\right\rangle_{l}=J(J+1)-\left\langle\hat{J}_{z}^{2}\right\rangle_{l}-\left\langle\hat{J}_{x}^{2}\right\rangle_{l} .
\end{gathered}
$$

The asterisk stands for the complex conjugate. Due to the thermalization of the electron-spin bath at a given temperature $T$, the square expectation values $\left\{\left\langle\hat{J}_{\alpha}^{2}\right\rangle_{l}\right\}_{l=1, \ldots,(2 J+1)(2 I+1) ; \alpha=x, y, z}$ of each $\alpha=x, y, z$ component are distributed according to the Boltzmann law. This defines the Boltzmann-averaged square expectation values ${\overline{\left\langle\hat{J}_{\alpha}\right.}}^{2}$ as

$$
\left\{{\overline{\left\langle\hat{J}_{\alpha}\right.}}^{2}=\sum_{l=1}^{(2 J+1)(2 I+1)} e^{-E_{l} / k_{B} T}\left\langle\hat{J}_{\alpha}^{2}\right\rangle_{l} / \sum_{l=1}^{(2 J+1)(2 I+1)} e^{-E_{l} / k_{B} T}\right\}_{\alpha=x, y, z} .
$$

This is the step where we adapt the model in Ref. [27], which estimates the contribution of a thermal nuclear-spin bath on the qubit dephasing rate, to estimate the contribution of a thermal electron-spin bath on the same rate. Indeed, in Ref. [27] each magnetic nucleus $i$ in the bath is described with an isotropic Zeeman Hamiltonian $\hat{H}_{i}=$ $\mu_{N} g_{N}^{i} \vec{B} \cdot \overrightarrow{I_{i}}$, where $\mu_{N}$ is the nuclear magneton, $g_{N}^{i}$ is the nuclear Landé factor, $\vec{B}$ is the applied magnetic field, and $\overrightarrow{I_{i}}$ is the nuclear-spin operator. The diagonalization of $\hat{H}_{i}$ leads to a set of energies and wave functions $\left\{E_{i, l},\left|\psi_{i, l}\right\rangle\right\}_{l=1, \ldots, 2 I_{i}+1}$, where $I_{i}$ is the ground nuclear-spin quantum number. These wave functions allow determining the square expectation values $\left\{\left\langle\hat{I}_{\alpha}^{2}\right\rangle_{i, l}=\left\langle\psi_{i, l}\left|\hat{I}_{\alpha}^{2}\right| \psi_{i, l}\right\rangle\right\}_{\alpha=x, y, z ; l=1, \ldots,\left(2 I_{i}+1\right)}$ of the $\alpha=$ $x, y, z$ components, which are also distributed according to the Boltzmann law at a given temperature $T$ and define $\left\{\left\langle{\overline{I_{\alpha}}}^{2}=\sum_{l=1}^{2 I_{i}+1} e^{-E_{i, l} / k_{B} T}\left\langle\hat{I}_{\alpha}^{2}\right\rangle_{i, l} / \sum_{l=1}^{2 I_{i}+1} e^{-E_{i, l} / k_{B} T}\right\}_{\alpha=x, y, z}\right.$. Since $\hat{H}_{i}$ is isotropic, each $\left\langle\hat{I}_{\alpha}^{2}\right\rangle_{i, l}$ must be independent of the magnetic-field direction. Thus, we can choose a direction at our convenience to calculate $\left\langle\hat{I}_{\alpha}^{2}\right\rangle_{i, l}$. By considering the magnetic field in the $Z$-axis direction, the wave functions $\left\{\left|\psi_{i, l}\right\rangle\right\}_{l=1, \ldots, 2 I_{i}+1}$ become $\left\{\left|m_{I_{i}}\right\rangle\right\}_{m_{I_{i}}=-I_{i}, \ldots, I_{i}}$, where $m_{I_{i}}$ are the several projections of $I_{i}$. Each $\left\langle\hat{I}_{\alpha}^{2}\right\rangle_{i, l}$ can now be easily calculated, resulting in $\left\langle m_{I_{i}}\left|\hat{I}_{z}^{2}\right| m_{I_{i}}\right\rangle=m_{I_{i}}^{2}$, while $\left\langle m_{I_{i}}\left|\hat{I}_{x}^{2}\right| m_{I_{i}}\right\rangle$ and $\left\langle m_{I_{i}}\left|\hat{I}_{y}^{2}\right| m_{I_{i}}\right\rangle$ are both equal to $\left(I_{i}\left(I_{i}+1\right)-m_{I_{i}}{ }^{2}\right) / 2$. At the working magnetic fields $|\vec{B}|<0.5 \mathrm{~T}$ and temperatures $T \geqslant$ $3 \mathrm{~K}$, each nuclear Zeeman energy $E_{i, l}$ is still far from reaching the thermal energy $k_{B} T$, thus $e^{-E_{i, l} / k_{B} T} \rightarrow 1$. Under this assumption, it is easy to prove that $\left\{\left\langle\hat{I}_{\alpha}\right\rangle_{i}=I_{i}\left(I_{i}+1\right) / 3\right\}_{\alpha=x, y, z}$, and the nuclear magnetic moment is defined as $\overrightarrow{m_{i}}=$ $\mu_{N} g_{N}^{i}\left(\overline{\left\langle\hat{I}_{x}\right\rangle_{i}}, \overline{\left\langle\hat{I}_{y}\right\rangle_{i}}, \overline{\left\langle\hat{I}_{z}\right\rangle_{i}}\right)$, where $\overline{\left\langle\hat{I}_{\alpha}\right\rangle_{i}}=\sqrt{{\overline{\left\langle\hat{I}_{\alpha}\right\rangle_{i}}}^{2}}$. In our case, we define the magnetic moments $\vec{m}_{j}$ and $\vec{m}_{k}$ of the magnetic metal ions $j, k$ in terms of the electron Landé factors $\left\{g_{\alpha}\right\}_{\alpha=x, y, z}$ and $\overline{\left\langle\hat{J}_{\alpha}\right\rangle}=\sqrt{{\overline{\left\langle\hat{J}_{\alpha}\right\rangle}}^{2}}$ as

$$
\vec{m}=\mu_{B}\left(g_{x} \overline{\left\langle\hat{J}_{x}\right\rangle}, g_{y} \overline{\left\langle\hat{J}_{y}\right\rangle}, g_{z} \overline{\left\langle\hat{J}_{z}\right\rangle}\right) .
$$

Note that both magnetic metal ions are modeled with the same crystal-field Hamiltonian in Eq. (1) and hence $\vec{m}_{j}=$ $\overrightarrow{m_{k}}$, which are renamed as $\vec{m}$. Moreover, in general terms the free-ion value $g$ in Eq. (1) will be used in the three Landé factors $\left\{g_{\alpha}\right\}_{\alpha=x, y, z}$. In Ref. [27] the thermal nuclearspin bath is coupled to a central spin qubit. Each one of the two qubit states, symmetric $S$ and antisymmetric $A$, generates a different dipolar magnetic field $\left\{\overrightarrow{B^{T}}\left(\vec{r}_{i}\right)\right\}_{T=S, A}$ at the position $\overrightarrow{r_{i}}$ of a given magnetic nucleus $i$ in the bath. Thus, two different dipolar interaction energies are involved: $\left\{E_{i}^{T}=\left(-\overrightarrow{B^{T}}\left(\overrightarrow{r_{i}}\right)\right) \cdot \vec{m}_{i}\right\}_{T=S, A}$. For a given qubit state either $S$ or $A$, the three terms $\left\{\left(-\overrightarrow{B^{T}}\left(\vec{r}_{i}\right)\right)_{\alpha}\left(\vec{m}_{i}\right)_{\alpha}\right\}_{\alpha=x, y, z}$ 
whose summation amounts to $E_{i}^{T}$ define the qubit-nucleus interaction vector as $\overrightarrow{E_{i}^{T}}=\left(\left(\overrightarrow{E_{i}^{T}}\right)_{x},\left(\overrightarrow{E_{i}^{T}}\right)_{y},\left(\overrightarrow{E_{i}^{T}}\right)_{z}\right) / 2$ with $\left\{\left(\overrightarrow{E_{i}^{T}}\right)_{\alpha}=\left(-\overrightarrow{B^{T}}\left(\overrightarrow{r_{i}}\right)\right)_{\alpha}\left(\vec{m}_{i}\right)_{\alpha}\right\}_{\alpha=x, y, z}$. The contribution of the magnetic nucleus $i$ to $E_{n}$ is the magnitude $\left\|\overrightarrow{E_{i}^{A}}-\overrightarrow{E_{i}^{S}}\right\|$ of the difference between these two qubit-nucleus interaction vectors. By considering the whole nuclear-spin bath, the square nuclear echo line half-width is found to be $E_{n}^{2}=$ $\sum_{i} \| \overrightarrow{E_{i}^{A}}-{\overrightarrow{E_{i}^{S}}}^{2}$.

Indeed, when the central spin qubit is in a given state $\alpha|0\rangle+\beta|0\rangle$ with $|\alpha|^{2}+|\beta|^{2}=1$, there exists an allowed set of $\prod_{i}\left(2 I_{i}+1\right)$ nuclear spin states in a finite nuclear-spin bath. For a large enough bath, the density of states as a function of energy converges to a Gaussian distribution with a Gaussian line shape by virtue of the so-called central limit theorem. The Gaussian line half-width is just $E_{n}$, and $E_{n}^{2}$ would be the corresponding variance associated with this distribution.
Nevertheless, there may be some special situations in which the nuclear density of states largely deviates from a Gaussianlike line. This can be encountered when all $\left\|\overrightarrow{E_{i}^{A}}-\overrightarrow{E_{i}^{S}}\right\|$ have similar enough values, or when one qubit-nucleus interaction dominates over the others. In this case, this model would not be valid anymore.

In our case, we propose to adapt this procedure to determine $E_{e}$ as follows. From the dipolar interaction energy $E_{j k}=-\overrightarrow{m_{k}} \cdot \overrightarrow{B_{j}}\left(\overrightarrow{r_{j k}}\right)$ in Eq. (3), we consider the three terms $\left\{-\left(\overrightarrow{m_{k}}\right)_{\alpha}\left(\overrightarrow{B_{j}}\left(\overrightarrow{r_{j k}}\right)\right)_{\alpha}\right\}_{\alpha=x, y, z}$ whose summation amounts to $E_{j k}$ and define the interaction vector between the magnetic metal ions $j, k$ as $\overrightarrow{E_{j k}}=\left(\left(\overrightarrow{E_{j k}}\right)_{x},\left(\overrightarrow{E_{j k}}\right)_{y},\left(\overrightarrow{E_{j k}}\right)_{z}\right) / 2$ with $\left\{\left(\overrightarrow{E_{j k}}\right)_{\alpha}=-\left(\overrightarrow{m_{k}}\right)_{\alpha}\left(\overrightarrow{B_{j}}\left(\overrightarrow{r_{j k}}\right)\right)_{\alpha}\right\}_{\alpha=x, y, z}$. We now define the contribution of the $j, k$ pair to $E_{e}$ as the magnitude $\left\|\overrightarrow{E_{j k}}\right\|$ which, after some algebra, reads as follows:

$$
\left\|\overrightarrow{E_{j k}}\right\|^{2}=\left(\frac{\mu_{0} \mu_{B}^{2}}{8 \pi r_{j k}^{3}}\right)^{2} \sum_{\alpha=x, y, z}\left[g_{\alpha} \overline{\left\langle\hat{J}_{\alpha}\right\rangle}\left(\left(1-3\left(\frac{\left(\overrightarrow{r_{j k}}\right)_{\alpha}}{r_{j k}}\right)^{2}\right) g_{\alpha} \overline{\left\langle\hat{J}_{\alpha}\right\rangle}-3 \frac{\left(\overrightarrow{r_{j k}}\right)_{\alpha}}{r_{j k}^{2}} \sum_{\substack{\beta=x, y, z \\ \beta \neq \alpha}}\left(\overrightarrow{r_{j k}}\right)_{\beta} g_{\beta} \overline{\left\langle\hat{J}_{\beta}\right\rangle}\right)\right]^{2} .
$$

To determine the square electron echo line half-width $E_{e}^{2}$, we add all $j, k$ pairs of magnetic metal ions and divide the result by the number $N$ of these ions in the bath as done elsewhere [26]:

$$
E_{e}^{2}=\frac{1}{N} \sum_{j=1}^{N-1} \sum_{k>j}^{N}\left\|\overrightarrow{E_{j k}}\right\|^{2} .
$$

As mentioned above, the molecule encoding the spin qubit consists of a single magnetic metal ion where several ligands are bonded to the ion. Depending on the overlapping degree between the ion and ligand orbitals, part of the spin density in the ion may be deployed towards the ligands and lead to an effective decrease in the electron-spin magnitude of the ion. This effect reduces the dipolar interaction between magnetic metal ions, and can be described by the so-called covalence parameter $0<\varepsilon \leqslant 1$ [26]. This parameter can be estimated by first-principles methods routinely employed in computational quantum chemistry such as those based on the so-called density-functional theory [31]. The limit value $\varepsilon=1$ corresponds to a spin density fully located at the magnetic metal ion. We incorporate $\varepsilon$ as a correction to $E_{n}$ and $E_{e}$. Thus, the collective phase memory time reads as $T_{m}^{n+e}=$ $\hbar \Delta /\left[\varepsilon^{2}\left(E_{n}^{2}+E_{e}^{2}\right)\right]$.

We will apply our method to a single crystal composed of many copies of $\mathbf{1}$. We model each copy of $\mathbf{1}$ with the same crystal-field Hamiltonian and hence the calculated $T_{m}^{n+e}$ will be the same for all of these copies. Nonetheless, our method can be readily extended to model experiments where a different crystal-field Hamiltonian is used to describe each magnetic molecule. For example, this situation can be encountered when there is a distribution of crystal-field parameters due to the presence of dislocations, defects, as well as other lattice strains in the crystal. In this case, one would first generate a sample of $M$ sets of parameters according to the given distribution. Then, $M$ decoherence times $\left(T_{m}^{n+e}\right)_{i}$ would be calculated in order to build an average spin echo $\sum_{i=1}^{M} \exp \left(-\tau /\left(T_{m}^{n+e}\right)_{i}\right) / M$. By plotting and fitting this average versus $\tau$ to a generalized exponential, we would obtain an overall decoherence time. This procedure can also be adapted for powder samples and frozen solutions. In this case, the overall spin echo would be additionally averaged over a set of different representative orientations of the magnetic molecule.

\section{RESULTS}

In system $\mathbf{1}, \mathrm{HoW}_{10}$, the spin anisotropy produced by the ligand coordination of the $\mathrm{Ho}^{\mathrm{III}}$ ion is described by the crystal-field Hamiltonian $\hat{H}=\sum_{k=2,4,6} B_{k}^{0} \hat{O}_{k}^{0}+B_{4}^{4} \hat{O}_{4}^{4}+$ $\vec{I} \cdot \boldsymbol{A} \cdot \vec{J}+\mu_{B} \vec{B} \cdot g \cdot \vec{J}$, with ground electron and nuclear spins $J=8$ and $I=7 / 2$ [4]. The ground $J=|L+S|$ multiplet of the $\mathrm{Ho}^{\mathrm{III}}$ ion arises from the spin-orbit coupled orbital and spin angular momenta $L=6$ and $S=2$. The crystal-field parameters are $B_{2}^{0}=0.601 \mathrm{~cm}^{-1}, B_{4}^{0}=6.96 \cdot 10^{-3} \mathrm{~cm}^{-1}$, $B_{6}^{0}=-5.10 \times 10^{-5} \mathrm{~cm}^{-1}, B_{4}^{4}=3.14 \times 10^{-3} \mathrm{~cm}^{-1}$ [4]. The hyperfine coupling parameters are $A_{z}=830 \mathrm{MHz}, A_{x}=A_{y}=$ 0 [4]. Since $\mathrm{Ho}^{\mathrm{III}}$ ion is described with its ground electron spin $J=8$, not as an effective spin $J=1 / 2, g$ is set to be the free-ion value of 1.25 [4]. We consider that all copies of $\mathbf{1}$ in the crystal have their magnetic anisotropy axis pointing in the same direction, which defines the direction of the applied magnetic field $\vec{B}$.

The axial terms $B_{2}^{0} \hat{O}_{2}^{0}, B_{4}^{0} \hat{O}_{4}^{0}, B_{6}^{0} \hat{O}_{6}^{0}$ in the crystal-field Hamiltonian result, in the case of $\mathbf{1}$, in an isolated $m_{J}= \pm 4$ 
ground doublet, separated from the first excited state (an $m_{J}=$ \pm 5 doublet) by about $20 \mathrm{~cm}^{-1}$. This is a robust result that has been confirmed repeatedly over the years by methods that rely on different assumptions on the crystal-field Hamiltonian and different experimental data, such as magnetic properties in the 2-300 K temperature range of a whole series of isostructural $\mathrm{Ln}^{\mathrm{III}} \mathrm{W}_{10}$ compounds [32,33], or a multifrequency EPR study of the ground electronuclear multiplet of $\mathbf{1}$ [34]. When combined with the nuclear-spin projections of $I=7 / 2$, the ground doublet $m_{J}= \pm 4$ gives rise to a low-lying multiplet of 16 states (Fig. 3). The sizable interaction $B_{4}^{4}$ generates an energy gap $\Delta \sim 9.18 \mathrm{GHz}$ at the ZEFOZ fields, which defines the long-lived spin qubit [4].

In Ref. [4] it was established that the extreme uniaxial anisotropy of $\mathbf{1}$ results in a rather negligible sensitivity to the perpendicular component of the applied magnetic field $\vec{B}$. In other words, the effective perpendicular Landé factor of the $m_{J}= \pm 4$ ground doublet is $g_{\perp \text {,eff }}<0.01$. Since the focus is on this doublet as it defines the spin qubit, the Zeeman term $\mu_{B} \vec{B} \cdot g \cdot \overrightarrow{\hat{J}}$ is replaced by the scalar approximation $\mu_{B} g B_{z} \hat{J}_{z}$, where the $z$ direction defines the molecular magnetic anisotropy axis and the direction of $\vec{B}$. Note that this does not mean that an axial $g$ factor is being used in $\hat{H}$ with $g=0$ in $\mu_{B} B_{x} g \hat{J}_{x}$ and $\mu_{B} B_{y} g \hat{J}_{y}$, while $g=1.25$ in $\mu_{B} B_{z} g \hat{J}_{z}$. Instead, the terms $\mu_{B} B_{x} g \hat{J}_{x}$ and $\mu_{B} B_{y} g \hat{J}_{y}$ are neglected after considering an isotropic factor $g=1.25$. The translation of this scalar approximation in terms of our Eq. (10) means to set $g_{z}=1.25$, $g_{x}=g_{y}=0$.

As lanthanide $4 f$ orbitals are internal enough not to deploy a significant spin density towards the ligands, we use $\varepsilon=$ 1 for 1. In Fig. 3, the qubit Zeeman curves have a slope approximately zero at the ZEFOZ fields, making $\Delta$ become insensitive to magnetic field up to first order [4]. This remarkably increases $T_{m}$, but only up to a maximum value. The $T_{m}^{n}$ calculation of at least $300 \mu$ s derived by applying the model in Ref. [27] to $\mathbf{1}$ is much above the experimental values. This agrees with the fact that magnetic nuclear decoherence might not be limiting qubit coherence at the ZEFOZ fields, given the clear experimental dependence of $T_{m}$ with the electron-spin concentration. Instead, our $T_{m}^{e}$ calculation, free of fitting parameters, excellently agrees with the maximum experimental $T_{m}$ values at the relevant electron-spin concentrations, which span over one order of magnitude (Fig. 3). In contrast, the model in Ref. [26] fails (Fig. 3). The high-field regime holds as the highest $E_{e} \sim 0.05 \mathrm{GHz} \ll \Delta \sim 9.18 \mathrm{GHz}$ (10\% concentration). Calculating $E_{e}$ in Eq. (11) requires knowing the Cartesian positions-necessary to evaluate Eq. (10)—of the magnetic metal ions in the molecular crystal. These positions have been determined by x-ray crystallography [4], and are provided as Supplemental Material [21].

Since the calculated $\overline{\left\langle\hat{J}_{x}\right\rangle}=\overline{\left\langle\hat{J}_{y}\right\rangle}=5.3, \overline{\left\langle\hat{J}_{z}\right\rangle}=4.0$ in Eq. (8) remain constant with $|\vec{B}|$ and are nonzero, $E_{e}^{2}$ in Eq. (11) also takes a nonzero value. Hence, $T_{m}^{e} \propto$ $1 / E_{e}^{2}$ cannot diverge and reach an arbitrarily high value at the ZEFOZ fields. Note that the analog of Eq. (10) in Ref. [27] is proportional to the expectation value differences $\left\{\left\langle\hat{J}_{\alpha}\right\rangle^{A}-\left\langle\hat{J}_{\alpha}\right\rangle^{S}\right\}_{\alpha=x, y, z}$ of the electron-spin operator components $\alpha=x, y, z$ calculated at the symmetric $S$ and antisym-
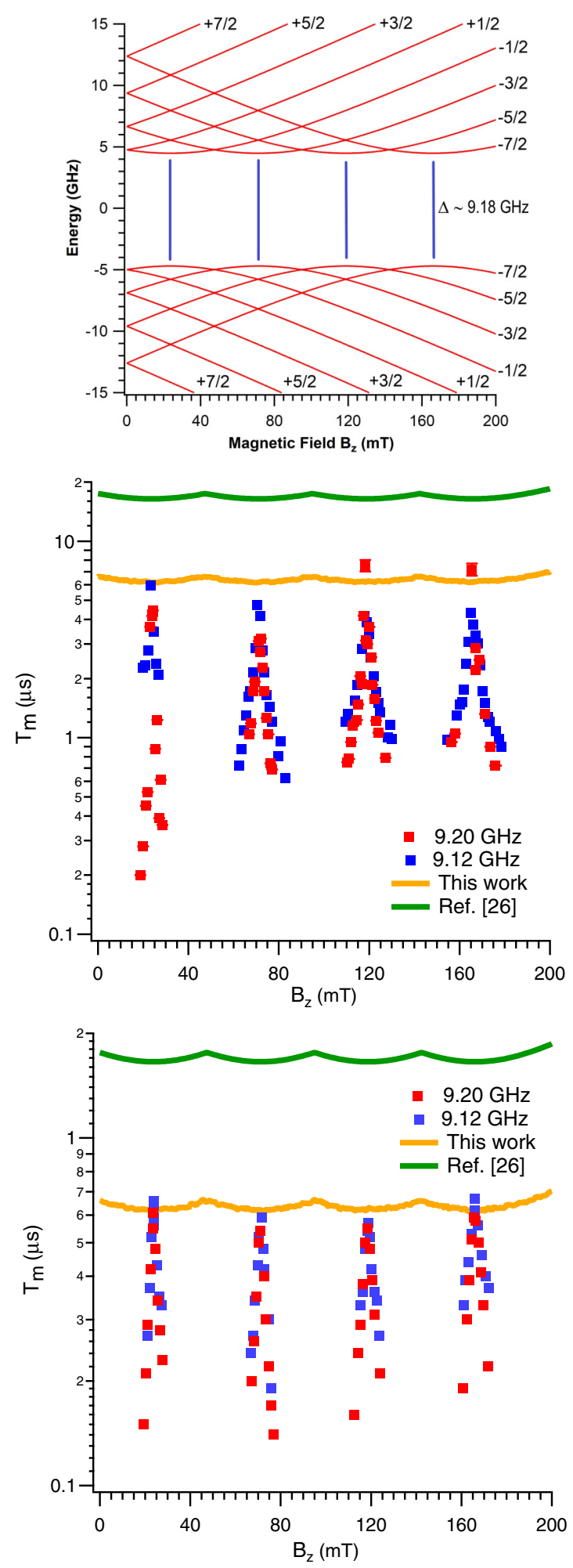

FIG. 3. Top: Zeeman energy evolutions of 1 labeled with the $m_{I}$ projections. Blue lines are ZEFOZ shifts. Middle and Bottom: magnetic-field dependence of $T_{m}$ (logarithmic scale) in $\mathbf{1}$ at $T=5 \mathrm{~K}$ for two electron-spin concentrations. Middle: 1\%. Bottom: $10 \%$. Blue and red points are experimental $T_{m}$ values at different microwave frequencies [4]. Green and orange curves are theoretical calculations using the model in Ref. [26]. and that of the present work, respectively. 
metric $A$ states of the qubit (see Supplemental Material 3 and 4 [21]) instead of $\left\{\overline{\left\langle\hat{J}_{\alpha}\right\rangle}\right\}_{\alpha=x, y, z}$ in Eq. (8). In a small neighborhood of the ZEFOZ fields, each $\left\langle\hat{J}_{\alpha}\right\rangle^{A}-\left\langle\hat{J}_{\alpha}\right\rangle^{S}$ tends to zero and $E_{n}^{2}$ vanishes. Thus, since $T_{m}^{n} \propto 1 / E_{n}^{2}$, the model in Ref. [27] predicts a divergence of the phase memory time at the ZEFOZ fields in contrast to the experimental behavior.

\section{DISCUSSION}

A striking feature of Fig. 3 is the small variation in the calculated $T_{m}^{e}$ with $|\vec{B}|$. This is because $T_{m}^{e} \propto \Delta / E_{e}^{2}$ and $E_{e}^{2}$ is constant in 1. The small rise in $T_{m}^{e}$ arises from the slight increase in $\Delta$ at fields away from the ZEFOZ fields. Our method is only capturing a certain decoherence mechanism in $T_{m}$, namely instantaneous diffusion [28,29], but neglects the main mechanism and thus does not recover the experimental $T_{m}$ decay. Once this main mechanism is suppressed at the ZEFOZ fields, instantaneous diffusion becomes the limiting relaxation process, which is confirmed given the experimentally observed stretching factor $x=1$, the noticeable dependence of $T_{m}$ with electron-spin concentration, and the match between experimental and calculated $T_{m}$ values. In fact, as stated in Ref. [4], ZEFOZ shifts are not able to protect the qubit from this specific relaxation process.

Moreover, the working conditions at the ZEFOZ fields, namely an experimental temperature of $5 \mathrm{~K}$ and a qubit gap $\Delta \sim 9.18 \mathrm{GHz}$, are among those ones for which the contribution of phonon decoherence to the EPR linewidth is rather negligible [11]. In fact, given the high electron-spin concentrations employed, it is at these conditions where electronspin-induced decoherence is limiting qubit coherence [11]. At $5 \mathrm{~K}$ in 1 , the spin-lattice relaxation time $T_{1}$ is about $20 \mu \mathrm{s}$. This experimentally determined parameter which decreases with temperature is importantly an upper bound of $T_{m}$, i.e., $T_{m} \leqslant T_{1}$. Thus, it is a figure employed to estimate how much vibration-related spin relaxation is affecting qubit coherence (the larger $T_{1}$ is, the larger $T_{m}$ could be). Its value of $20 \mu \mathrm{s}$ at $5 \mathrm{~K}$ is close to the reported $T_{m}$ values, but yet above enough not to reasonably expect that phonon decoherence is limiting qubit coherence, especially given the high electronspin concentrations employed.

Whenever instantaneous diffusion dominates, tuning $T_{m}^{e}$ needs to properly engineer $\Delta$ and $E_{e}^{2}$. The former depends on the electronic structure determined by Eq. (1), and the latter depends additionally on the electron-spin concentration in the sample. The qubit gap $\Delta$ in $\mathbf{1}$ at the ZEFOZ fields is set by the $B_{4}^{4}$ parameter, which is activated because of the deviation from the $D_{4 d}$ symmetry in the Ho-coordinating oxygen atoms set [4]. Simple calculations reveal that $\Delta$ scales with $B_{4}^{4}$ as $\Delta(\mathrm{GHz})=2.0 B_{4}^{4}\left(\mathrm{~cm}^{-1}\right)+926900\left(B_{4}^{4}\right)^{2}\left(\mathrm{~cm}^{-2}\right)$. Besides, Eq. (8) is unaffected by changing $B_{4}^{4}$ in a wide range around its value $3.14 \times 10^{-3} \mathrm{~cm}^{-1}$. Thus, given an electronspin concentration, we expect that a rise in $B_{4}^{4}$ will increase $\Delta$ while keeping $E_{e}^{2}$ unaltered. Since $T_{m}^{e} \propto \Delta / E_{e}^{2}$, the phase memory should be consequently increased.

To understand how $T_{m}^{e}$ scales with electron-spin concentration, let us fix the electronic structure with given values of $\Delta, g_{\alpha}$ and Eq. (8). If we replace $r_{j k}$ in Eq. (10) by an average effective distance, $E_{e}^{2}$ becomes proportional to
$(1 / N) N(N-1) / 2$. Since $N$ is large, $T_{m}^{e} \propto 1 / N$, as also recently stated [35]. To assess the validity of this expression, let us recall that $T_{m}$ in $\mathbf{1}$ is tenfold larger as electron-spin concentration decreases by one order of magnitude. Thus, we expect $N_{10 \%} / N_{1 \%}=10$. Indeed, the $E_{e}^{2}$ calculation is converged with $N_{10 \%}=88259, N_{1 \%}=8832$ (see Supplemental Material 1 and 2 [21]), and $N_{10 \%} / N_{1 \%}=9.993$. In summary, our method successfully reproduces the experimental $T_{m}$ top values at the ZEFOZ fields in $\mathbf{1}$, and provides insight on the factors limiting qubit coherence (see also below).

As a further check we also applied our method to 2 , CuPc [26], and found that it is also able to reproduce the experimental $T_{m}$ evolution of $\mathbf{2}$ as a function of electronspin concentration. $\mathbf{2}$ is a magnetic molecule whose qubit is encoded in the ground electron spin $J=S=1 / 2$ of a single $\mathrm{Cu}^{2+}$ ion coupled to its ground nuclear spin $I=3 / 2$. The energy-level scheme is composed of eight spin states, which arise from the spin Hamiltonian $\hat{H}=\overrightarrow{\hat{I}} \cdot \boldsymbol{A} \cdot \overrightarrow{\hat{J}}+\mu_{B} \vec{B}$ $\mathrm{g} \cdot \overrightarrow{\vec{J}}$. The copper electron-nuclear hyperfine coupling is set by $A_{z}=-648 \mathrm{MHz}, A_{x}=A_{y}=-83 \mathrm{MHz}$, and the Zeeman effect is due to an external field $|\vec{B}|=311.5 \mathrm{mT}$, with $g_{z}=$ 2.1577, $g_{x}=g_{y}=2.0390$. The perpendicular direction to the molecular plane (see Supplemental Material 2 [21]) corresponds to the magnetic anisotropy axis of $\mathbf{2}$, which defines the direction of the applied magnetic field $\vec{B}$. The transition $\left|m_{J}=-1 / 2, m_{I}=-1 / 2\right\rangle \rightarrow\left|m_{J}=+1 / 2, m_{I}=-1 / 2\right\rangle$ defines the qubit, with a gap $\Delta \sim 9.73 \mathrm{GHz}$. Note that whenever the magnetic metal ion is treated as a ground electron spin $J=1 / 2$ - either real or effective - the crystal-field terms disappear from the Hamiltonian in Eq. (1), and the electron Landé factors $g_{x}, g_{y}, g_{z}$ are now varied to fit the given cw-EPR spectrum. These fitting values, which may differ from the free-ion value, are used in Eq. (10) to calculate $T_{m}^{e}$.

The main contribution to $T_{m}$ in $\mathbf{2}$ from the nuclear-spin bath is due to the nuclei of the four copper-coordinating nitrogen atoms in Fig. S1 [26]. The interaction between the copper ion and these nuclei corresponds to a contact hyperfine coupling, much stronger than a magnetic dipolar interaction, with coupling constants $A_{x x}^{N}=57 \mathrm{MHz}, A_{y y}^{N}=A_{z z}^{N}=45 \mathrm{MHz}$ (see Supplemental Material [21]). By setting $\varepsilon=0.74$, a nuclear phase memory time of $T_{m}^{n}=2.2 \mu s$ is obtained [26]. Nitrogen nuclei are considered to have nuclear spin $I_{N}=1$, since this corresponds to the most occurring isotope. The high-field approximation holds as $E_{n} \sim 0.03 \mathrm{GHz} \ll \Delta \sim 9.73 \mathrm{GHz}$.

In a second calculation the rest of the nuclear-spin bath is added, and it is found that the calculated $T_{m}^{n}$ value remains unchanged. This fact confirms that the nuclear-spin-bath contribution to $T_{m}$ is limited by the nuclei of the four coppercoordinating nitrogen atoms. This is most likely due to the direct and very strong hyperfine coupling with the nitrogen nuclei due to their proximity to the copper ion, and the fact that the closest hydrogen nuclei are more than $5.5 \AA$ away. This extreme proximity of magnetic nuclei to the magnetic metal ion encoding the qubit is not present in $\mathbf{1}$. In this system, the $\mathrm{Ho}^{3+}$ ion is coordinated by eight oxygen atoms, and their most occurring isotope ( ${ }^{16} \mathrm{O}$ with a $99.8 \%$ occurrence) has a nonmagnetic nucleus. Hence, magnetic nuclear decoherence is reasonably expected not to play an important role, especially given the high electron-spin concentrations employed. 


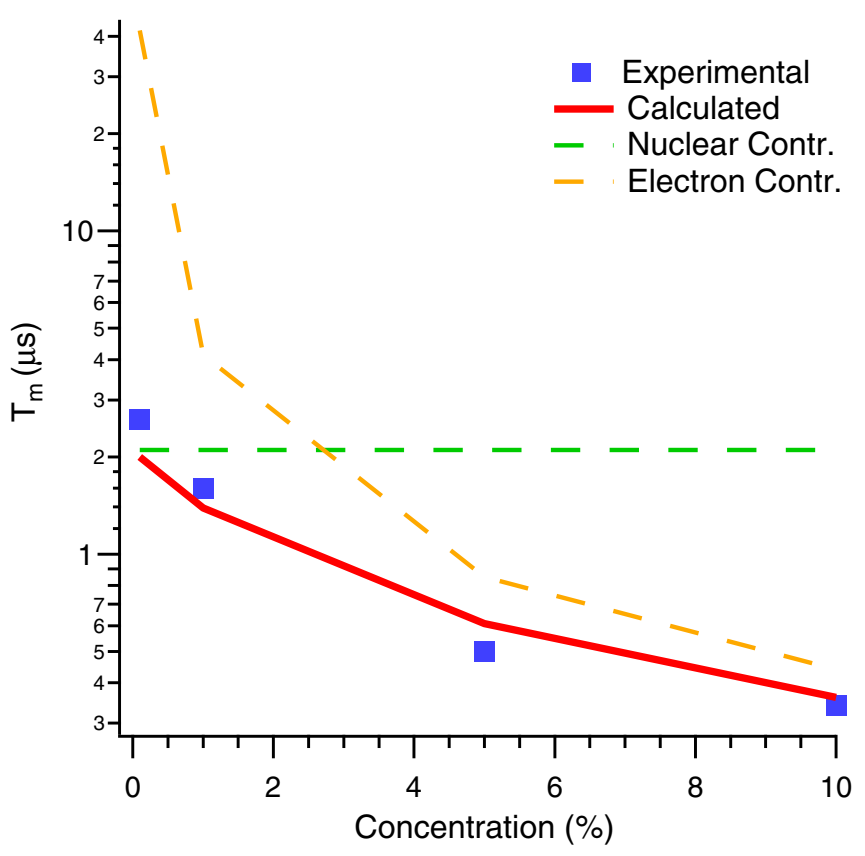

FIG. 4. Phase memory time evolution (logarithmic scale) of $\mathbf{2}$ at $T=5 \mathrm{~K}$ with the electron-spin concentration. The experimental blue points are from Ref. [26]. Green and orange dashed lines are the nuclear- and electron-spin-bath contributions to $T_{m}^{n+e}$ (red curve) calculated by our method.

Note that this mechanism in $\mathbf{2}$ is only determining at low enough electron-spin concentration (Fig. 4).

Calculating $E_{e}$ in 2 also requires the Cartesian positions of the magnetic metal ions (here, $\mathrm{Cu}^{2+}$ ions) in the electronspin bath, which is limited to a spherical granule of 50-nm diameter [26] (see Supplemental Material 2 [21] and Fig. S1). These position - required to evaluate Eq. (10)—are provided as Supplemental Material [21]. The high-field approximation holds again as the highest $E_{e} \sim 0.06 \mathrm{GHz} \ll \Delta \sim 9.73 \mathrm{GHz}$ (10\% concentration), and the calculated nuclear-electron $T_{m}^{n+e}$ values with $\varepsilon=0.74$ satisfactorily agree with the experiment (Fig. 4). As expected, electron-spin-induced decoherence dominates at high electron-spin concentration, while nuclear-spin-induced decoherence dominates at low concentration. Note that we are able to reproduce the experimental $T_{m}$ values in $\mathbf{2}$ even though the working applied magnetic field does not correspond to any ZEFOZ field. Indeed, exponentials with stretching factor $x=1$ were satisfactorily used to fit the echo decays at high electron-spin concentration in 2 [26], which indicates that instantaneous diffusion is the dominant dephasing process. Moreover, $T_{1}$ is now at least two orders of magnitude above $T_{m}$. Hence, phonon-induced decoherence might have even a lesser influence than in system 1.

To end, it is interesting to unveil additional factors that can make $T_{m}$ values be different between protected systems via ZEFOZ shifts 1 and simple $S=1 / 2$ systems 2 once instantaneous diffusion becomes the limiting decoherence mechanism, namely, at high enough electron-spin concentrations. Indeed, at the highest electron-spin concentration, the $T_{m}^{\mathrm{e}}$ values in $\mathbf{1}$ at the ZEFOZ fields are appreciably higher than that of 2 (Figs. 3 and 4). To figure this difference out, let us fix an $8.2 \%$ concentration in $\mathbf{1}$, equivalent to $10 \%$ in $\mathbf{2}$ (see Supplemental Material 1 and 2 [21]). The Eq. (8) values in 2 are $\overline{\left\langle\hat{J}_{x}\right\rangle}=\overline{\left\langle\hat{J}_{y}\right\rangle}=\overline{\left\langle\hat{J}_{z}\right\rangle}=0.5$, much smaller than those of 1. As $E_{e}^{2}$ is initially proportional to $\left\{{\overline{\left\langle\hat{J}_{\alpha}\right\rangle}}_{\alpha=x, y, z}\right.$, Eq. (10), we would expect a greater $T_{m}^{e}$ value in 2 .

To explain the rather opposite behavior, we need to focus on the electron Landé factors of $\mathbf{1}$ and $\mathbf{2}$. While all the electron Landé factors $\left\{g_{\alpha}\right\}_{\alpha=x, y, z}$ are nonzero in $\mathbf{2}$, only $g_{z}$ is different from zero in $\mathbf{1}$. Since Eq. (10) is a three-term and non-negative sum proportional to $g_{\alpha}, E_{e}^{2}$ takes a smaller value for 1 , which results in a larger $T_{m}^{e}$ value since $T_{m}^{e} \propto 1 / E_{e}^{2}$ for a similar qubit gap $\Delta$. Indeed, simple calculations show that a decrease of $g_{x}$ and $g_{y}$ in $\mathbf{2}$ raises $T_{m}^{e}$. Let us recall that the extreme uniaxial anisotropy of $\mathbf{1}$ makes the perpendicular component of the effective electron Landé tensor associated to the ground doublet $m_{J}= \pm 4$ be negligible, which allows using $g_{x}=g_{y}=0$ in Eq. (10). This reveals the crucial role of having an axial electron Landé tensor, with rather negligible perpendicular components and a small parallel component, corresponding to the spin doublet-either real or effectivethat defines the qubit. Thus, depending on the system, finetuning of the electron Landé $g_{\alpha}$ factors that characterize the qubit spin doublet may be required to maximize $T_{m}$.

Herein, we have proposed a method free of fitting parameters that satisfactorily simulates the influence of a high electron-spin concentration on the phase memory time $T_{m}$ of a given spin qubit. The method relies on a model devoted to estimate the contribution of magnetic nuclear decoherence to $T_{m}$ [27], and works provided instantaneous diffusion (ID) is the dominant decoherence mechanism. This method is valid both in isotropic and anisotropic magnetic systems, and can deal with distributions of the electron structure parameters in Eq. (1). Note that the employed parameters in Eq. (1) characterizing systems $\mathbf{1}$ and $\mathbf{2}$ were determined from cw-EPR experiments. Whenever experimental data are not available, one can resort to first-principles codes that are commonly employed in computational quantum chemistry to estimate these parameters (e.g., MOLCAS and ORCA) [36,37]. Our method accounts for qubit coherence in challenging systems displaying ZEFOZ shifts where recent models fail [26], but is not intrinsically tied to clock transitions. Indeed, in the case of $\mathbf{1}$, ID is the dominant mechanism at the clock transitions, while other relaxation mechanisms dominate at fields away from these avoided crossings. Thus, the calculated phase memory times at these fields are overestimated. In the case of $\mathbf{2}$, the method correctly reproduces the experimental phase memory times for a regular (not clock) transition, which indicates that for this system and at the working conditions the main relaxation mechanism is ID at the explored electron-spin concentrations. Our results state that a properly engineered electronic structure can result in enhanced ZEFOZ coherences. Namely, important focus should be put on (i) the electron Landé tensor of the spin doublet-either real or effective-that defines the qubit, which should be axial with negligible perpendicular components and a small parallel component; (ii) key parameters that increase the qubit energy gap. Hence, this method constitutes a widely applicable tool able to offer insight on understanding decoherence towards 
integrating spin qubits in quantum devices. Because of the potential applicability of ZEFOZ-based approaches in providing close proximity and coherent spin qubits, in a future work we will address the issue of the fast $T_{m}$ decay out of the ZEFOZ fields.

The authors declare that the data supporting the findings of this study are available within the paper and its Supplemental Material files.

\section{ACKNOWLEDGMENTS}

The present work has been funded by the EU (COST Actions No. CA15128 MOLSPIN, No. ERC-2014-CoG-647301
DECRESIM, and No. ERC-2018-AdG-788222 MOL-2D), the Spanish MINECO (Unit of excellence "María de Maeztu" No. MDM-2015-0538 and Grants No. MAT2017-89993$\mathrm{R}$ and No. CTQ2017-89528-P) and the Generalitat Valenciana (Prometeo Program of excellence). L.E.-M. thanks also the Generalitat Valenciana for a VALi+d predoctoral contract and A.G.-A. acknowledges funding by the MINECO (Ramón y Cajal contract). We thank Dr. I. S. Tupitsyn for fruitful discussions. L.E.-M. developed the theory, performed the calculations, and analyzed the results. A.G.A. and E.C. helped L.E.-M. to write the manuscript. All authors discussed and reviewed the final version of the manuscript.

The authors declare no competing interests.
[1] R. Hanson, V. V. Dobrovitski, A. E. Feiguin, O. Gywat, and D. D. Awschalom, Coherent dynamics of a single spin interacting with an adjustable spin bath, Science 320, 352 (2008).

[2] M. Steger, K. Saeedi, M. L. W. Thewalt, J. J. L. Morton, H. Riemann, N. V. Abrosimov, P. Becker, and H.-J. Pohl, Quantum information storage for over $180 \mathrm{~s}$ using donor spins in a ${ }^{28} \mathrm{Si}$ "Semiconductor vacuum", Science 336, 1280 (2012).

[3] J. M. Zadrozny, J. Niklas, O. G. Poluektov, and D. E. Freedman, Millisecond coherence time in a tunable molecular electronic spin qubit, ACS Cent. Sci. 1, 488 (2015).

[4] M. Shiddiq, D. Komijani, Y. Duan, A. Gaita-Ariño, E. Coronado, and S. Hill, Enhancing coherence in molecular spin qubits via atomic clock transitions, Nature (London) 531, 348 (2016).

[5] C. Monroe and J. Kim, Scaling the ion trap quantum processor, Science 339, 1164 (2013).

[6] M. H. Devoret and R. J. Schoelkopf, Superconducting circuits for quantum information: An outlook, Science 339, 1169 (2013)

[7] L. Escalera-Moreno, N. Suaud, A. Gaita-Ariño, and E. Coronado, Determining key local vibrations in the relaxation of molecular spin qubits and single-molecule magnets, J. Phys. Chem. Lett. 8, 1695 (2017).

[8] L. Escalera-Moreno, J. J. Baldoví, A. Gaita-Ariño, and E. Coronado, Spin states, vibrations and spin relaxation in molecular nanomagnets and spin qubits: A critical perspective, Chem. Sci. 9, 3265 (2018).

[9] A. Lunghi, F. Totti, R. Sessoli, and S. SanvitoThe role of anharmonic phonons in under-barrier spin relaxation of single molecule magnets, Nat. Commun. 8, 14620 (2017).

[10] C. A. P. Goodwin, F. Ortu, D. Reta, N. F. Chilton, and D. P. Mills, Molecular magnetic hysteresis at 60 kelvin in dysprosocenium, Nature (London) 548, 439 (2017).

[11] S. Takahashi, I. S. Tupitsyn, J. van Tol, C. C. Beedle, D. N. Hendrickson, and P. C. E. Stamp, Decoherence in crystals of quantum molecular magnets, Nature (London) 476, 76 (2011).

[12] K. Bader, D. Dengler, S. Lenz, B. Endeward, S.-D. Jiang, P. Neugebauer, and J. van Slageren, Room temperature quantum coherence in a potential molecular qubit, Nat. Commun. 5, 5304 (2014).

[13] J. Ferrando-Soria et al., A modular design of molecular qubits to implement universal quantum gates, Nat. Commun. 7, 11377 (2016).
[14] F. Luis et al., Molecular Prototypes for Spin-Based CNOT and SWAP Quantum Gates, Phys. Rev. Lett. 107, 117203 (2011).

[15] D. Aguilà, L. A. Barrios, V. Velasco, O. Roubeau, A. Repollés, P. J. Alonso, J. Sesé, S. J. Teat, F. Luis, and G. Aromí, Heterodimetallic [ $\left.\mathrm{LnLn}^{\prime}\right]$ lanthanide complexes: Toward a chemical design of two-qubit molecular spin quantum gates, J. Am. Chem. Soc. 136, 14215 (2014).

[16] G. Wolfowicz, A. M. Tyryshkin, R. E. George, H. Riemann, N. V. Abrosimov, P. Becker, H.-J. Pohl, M. L. W. Thewalt, S. A. Lyon, and J. J. L. Morton, Atomic clock transitions in siliconbased spin qubits, Nat. Nanotechnol. 8, 561 (2013).

[17] E. Fraval, M. J. Sellars, and J. J. Longdell, Method of Extending Hyperfine Coherence Times in $\mathrm{Pr}^{3+}: \mathrm{Y}_{2} \mathrm{SiO}_{5}$, Phys. Rev. Lett. 92, 077601 (2004).

[18] M. Zhong, M. P. Hedges, R. L. Ahlefeldt, J. G. Bartholomew, S. E. Beavan, S. M. Wittig, J. J. Longdell, and M. J. Sellars, Optically addressable nuclear spins in a solid with a six-hour coherence time, Nature (London) 517, 177 (2015).

[19] J. M. Zadrozny, A. T. Gallagher, T. D. Harris, and D. E Freedman, A porous array of clock qubits, J. Am. Chem. Soc. 139, 7089 (2017).

[20] M. D. Jenkins, D. Zueco, O. Roubeau, G. Aromí, J. Majer, and F. Luis, A scalable architecture for quantum computation with molecular nanomagnets, Dalton Trans. 45, 16682 (2016).

[21] See Supplemental Material at http://link.aps.org/supplemental/ 10.1103/PhysRevB.100.064405 for details about generating spin baths and simulating spin concentrations (Secs. 1 and 2), the nuclear-spin-induced decoherence model (Secs. 3 and 4), characteristic features of our method as compared to previous models (Sec. 5), details on molecular spin qubits working at atomic clock transitions (Sec. 6), and to access the spin-bath positions determined by $\mathrm{x}$-ray crystallography. The Supplemental Material also includes Refs. [22-25].

[22] C. Godfrin, A. Ferhat, R. Ballou, S. Klyatskaya, M. Ruben, W. Wernsdorfer, and F. Balestro, Operating Quantum States in Single Magnetic Molecules: Implementation of Grover's Quantum Algorithm, Phys. Rev. Lett. 119, 187702 (2017).

[23] C. Bonizzoni, A. Ghirri, and M. Affronte, Coherent coupling of molecular spins with microwave photons in planar superconducting resonators, Adv. Phys.: X 3, 1435305 (2018).

[24] C. Bonizzoni, A. Ghirri, M. Atzori, L. Sorace, R. Sessoli, and M. Affronte, Coherent coupling between Vanadyl Phthalocyanine spin ensemble and microwave photons: towards integration 
of molecular spin qubits into quantum circuits, Sci. Rep. 7, 13096 (2017).

[25] A. Gaita-Ariño, F. Luis, S. Hill, and E. Coronado, Molecular spins for quantum computation, Nat. Chem. 11, 301 (2019).

[26] M. Warner et al., Potential for spin-based information processing in a thin-film molecular semiconductor, Nature (London) 503, 504 (2013).

[27] P. C. E. Stamp and I. S. Tupitsyn, Coherence window in the dynamics of quantum nanomagnets, Phys. Rev. B 69, 014401 (2004).

[28] A. Schweiger and G. Jeschke, Principles of Pulse Electron Paramagnetic Resonance (Oxford University Press, Oxford, 2001).

[29] Distance Measurements in Biological Systems by EPR, edited by L. J. Berliner, S. S. Eaton, and G. R. Eaton (Springer, New York, 2000).

[30] C. Rudowicz and C. Y. Chung, The generalization of the extended Stevens operators to higher ranks and spins, and a systematic review of the tables of the tensor operators and their matrix elements, J. Phys.: Condens. Matter 16, 5825 (2004).

[31] C. Finazzo, C. Calle, S. Stoll, S. van Doorslaer, and A. Schweiger, Matrix effects on copper(II)phthalocyanine complexes. A combined continuous wave and pulse EPR and DFT study, Phys. Chem. Chem. Phys. 8, 1942 (2006).
[32] M. A. AlDamen, S. Cardona-Serra, J. M. Clemente-Juan, E. Coronado, A. Gaita-Ariño, C. Martí-Gastaldo, F. Luis, and $\mathrm{O}$. Montero, Mononuclear lanthanide single molecule magnets based on the polyoxometalates $\left[\mathrm{Ln}\left(\mathrm{W}_{5} \mathrm{O}_{18}\right)_{2}\right]^{9^{-}}$and $\left[\mathrm{Ln}\left(\beta_{2}-\mathrm{SiW}_{11} \mathrm{O}_{39}\right)_{2}\right]^{13-}\left(\mathrm{Ln}^{\mathrm{III}}=\mathrm{Tb}, \mathrm{Dy}, \mathrm{Ho}, \mathrm{Er}, \mathrm{Tm}, \mathrm{Yb}\right)$, Inorg. Chem. 48, 3467 (2009).

[33] J. J. Baldoví, J. M. Clemente-Juan, E. Coronado, Y. Duan, A. Gaita-Ariño, and C. Giménez-Saiz, Construction of a general library for the rational design of nanomagnets and spin qubits based on mononuclear f-block complexes. the polyoxometalate case, Inorg. Chem. 53, 9976 (2014).

[34] S. Ghosh, S. Datta, L. Friend, S. Cardona-Serra, A. Gaita-Ariño, E. Coronado, and S. Hill, Multi-frequency EPR studies of a mononuclear holmium single-molecule magnet based on the polyoxometalate $\left[\mathrm{Ho}^{\mathrm{III}}\left(\mathrm{W}_{5} \mathrm{O}_{18}\right)_{2}\right]^{9^{-}}$, Dalton Trans. 41, 13697 (2012).

[35] A. Lunghi and S. Sanvito, Electronic spin-spin decoherence contribution in molecular qubits by quantum unitary spin dynamics, J. Magn. Magn. Mater. 487, 165325 (2019).

[36] F. Aquilante et al., MOLCAS 8: New capabilities for multiconfigurational quantum chemical calculations across the Periodic Table, J. Comput. Chem. 37, 506 (2016).

[37] F. Neese, The ORCA program system, Wiley Interdiscip. Rev. Comput. Mol. Sci. 2, 73 (2012). 